\title{
Geometry and dynamics of some meromorphic functions
}

\author{
Janina Kotus ${ }^{* 1}$ and Mariusz Urbański $* * 2$ \\ ${ }^{1}$ Faculty of Mathematics and Information Sciences, Warsaw University of Technology, Warsaw 00-661, Poland \\ ${ }^{2}$ Department of Mathematics, University of North Texas, P.O. Box 311430, Denton, TX 76203-1430, USA
}

Received 2 April 2004, revised 11 January 2005, accepted 7 October 2005

Published online 7 September 2006

Key words Meromorphic function, Julia set, Hausdorff dimension, packing measure, invariant measure MSC (2000) Primary: 37F35; Secondary: 37F10, 30D05

The meromorphic maps $f_{\lambda}(z)=\lambda(1-\exp (-2 z))^{-1}, \lambda>0$, of the complex plane are thoroughly investigated. With each map $f_{\lambda}$ associated is its projection $F_{\lambda}$ on the infinite cylinder $Q$. This map and the set $J_{r}\left(F_{\lambda}\right)$ consisting of those points in the cylinder $Q$ whose $\omega$-limit set under $F_{\lambda}$ is not contained in the set $\{0,-\infty\}$ will form the primary objects of our interest in this article. Let $h_{\lambda}=\operatorname{HD}\left(J_{r}\left(F_{\lambda}\right)\right)$ be the Hausdorff dimension of $J_{r}\left(F_{\lambda}\right)$. We prove that $h_{\lambda} \in(1,2)$. The $h_{\lambda}$-dimensional Hausdorff measure $\mathrm{H}_{h_{\lambda}}$ of $J_{r}\left(F_{\lambda}\right)$ is proven to be positive and finite. The $h_{\lambda}$-dimensional packing measure of $J_{r}\left(F_{\lambda}\right)$ is shown to be locally infinite at every point of this set. There exists a unique Borel probability $F_{\lambda}$-invariant measure $\mu_{\lambda}$ on $J_{r}\left(F_{\lambda}\right)$ absolutely continuous with respect to the Hausdorff measure $\mathrm{H}_{h_{\lambda}}$. This measure turns out to be ergodic and equivalent to $\mathrm{H}_{h_{\lambda}}$.

(c) 2006 WILEY-VCH Verlag GmbH \& Co. KGaA, Weinheim

\section{Introduction}

The Fatou set $F(f)$ of a meromorphic function $f: \mathbb{C} \rightarrow \overline{\mathbb{C}}$ is the set of all points $z \in \mathbb{C}$ such that all the iterates are well-defined and form a normal family on a neighborhood of $z$. The Julia set $J(f)$ is the complement of $F(f)$ in $\mathbb{C}$. Thus, the Fatou set is open whereas $J(f)$ is closed, the Fatou set is completely invariant, while $f^{-1}(J(f)) \subset J(f)$ and $f(J(f)) \subset J(f) \cup\{\infty\}$. This latter property enables us to consider the dynamical system $f: J(f) \rightarrow J(f) \cup\{\infty\}$. For a general description of the topological dynamics of meromorphic functions the reader may consult [1]-[5]. It follows from Montel's criterion of normality that if $f: \mathbb{C} \rightarrow \overline{\mathbb{C}}$ has at least one pole which is not an omitted value then

$$
J(f)=\overline{\bigcup_{n \geq 1} f^{-n}(\infty)} .
$$

In this paper we continue the extensive study (see [16]-[19] and [29]-[32]) of the geometric and dynamical structure of the Julia sets of transcendental entire and meromorphic functions. Namely, given $\lambda>0$ we consider the function

$$
f_{\lambda}(z)=\frac{\lambda}{1-\exp (-2 z)}
$$

The research presented in this article stems from [29] and [32]. In order to give an overview of what our paper is about, let us introduce first the equivalence relation $\sim$ in $\mathbb{C}$ by saying that $w \sim z$ if and only if $w-z \in \pi i \mathbb{Z}$. We then define the cylinder

$$
Q:=\mathbb{C} / \sim
$$

and let

* e-mail: janinak@impan.gov.pl

** Corresponding author: e-mail: urbanski@unt.edu, web: http://www.math.unt.edu/ urbanski 


$$
\Pi: \mathbb{C} \longrightarrow Q
$$

be the quotient map. Since each function $f_{\lambda}$ is $\pi i$-periodic, it induces a unique meromorphic map $F_{\lambda}: Q \backslash\{0\} \rightarrow$ $Q$ such that $\Pi \circ f_{\lambda}=F_{\lambda} \circ \Pi$. In order to simplify notation we declare $F_{\lambda}(0)=\infty$ so that we may consider the function

$$
F_{\lambda}: Q \longrightarrow Q \cup\{\infty\} .
$$

Observe that 0 is a discontinuity point of $F_{\lambda}$. Note that $\Pi \circ f_{\lambda}^{n}=F_{\lambda}^{n} \circ \Pi$ for every integer $n \geq 1$, that

$$
J\left(F_{\lambda}\right)=\Pi\left(J\left(f_{\lambda}\right)\right)
$$

and the map $F_{\lambda}$ reflects many interesting features of the dynamics of $f_{\lambda}$, for example like escaping to $-\infty$. The subset $J_{r}\left(F_{\lambda}\right)$ of $F_{\lambda}$ is defined to consists of all points in $Q$ whose $\omega$-limit set under $F_{\lambda}$ is different from the set $\{0,-\infty\}$. The map $F_{\lambda}$ and the set $J_{r}\left(F_{\lambda}\right)$ will form the primary objects of our interest in this article. Let $h_{\lambda}=\operatorname{HD}\left(J_{r}\left(F_{\lambda}\right)\right)$ be the Hausdorff dimension of $J_{r}\left(F_{\lambda}\right)$. The main results of our paper are those

- $h_{\lambda} \in(1,2)$,

- the $h_{\lambda}$-dimensional Hausdorff measure $\mathrm{H}_{h_{\lambda}}$ of $J_{r}\left(F_{\lambda}\right)$ is positive and finite,

- the $h_{\lambda}$-dimensional packing measure of $J_{r}\left(F_{\lambda}\right)$ is locally infinite at every point of this set,

- there exists a unique Borel probability $F_{\lambda}$-invariant measure $\mu_{\lambda}$ on $J_{r}\left(F_{\lambda}\right)$ absolutely continuous with respect to the Hausdorff measure $\mathrm{H}_{h_{\lambda}}$. This measure is ergodic and equivalent to $\mathrm{H}_{h_{\lambda}}$.

As the reader sees, these four results constitute the basic geometrical and dynamical properties of the set $J_{r}\left(F_{\lambda}\right)$ and the map $F_{\lambda}: J_{r}\left(F_{\lambda}\right) \rightarrow J_{r}\left(F_{\lambda}\right)$, the properties one is always tempted to establish or to disprove (see [16]-[19] and [29]-[32]) when dealing with dynamical systems and its invariant fractal sets.

Let us now present briefly, though in greater detail, the contents of this article and the methods used. In Section 3 we introduce the sets $K_{M}$ and we prove that for all $M>0$ large enough their Hausdorff dimension is larger than 1, the fact which is absolutely necessary for all further results of this paper to be provable. In particular, in Section 3 we prove that the Hausdorff dimension of the set $J_{b d}\left(F_{\lambda}\right)$ is larger than one. In Section 4 equipped with the map $F_{\lambda}$, the concept of tightness, and the $K(V)$ method from [10] (comp. [23, Ch. 10]), we prove the existence and uniqueness of a Borel probability conformal measure $m$ (with an exponent greater than 1 ) for the map $F_{\lambda}$. We also prove there that the conformal measure is supported on $J_{r}\left(F_{\lambda}\right)$. In Section 5 we establish the existence of a unique Borel ergodic $F_{\lambda}$-invariant measure equivalent to the conformal measure. We do this by applying first the method of Marco Martens to show the existence of a $\sigma$-finite $F_{\lambda}$-invariant conservative ergodic measure equivalent to the measure $m$ and checking then that this measure is finite. The Section 6 is occupied with geometric issues. We prove there that the packing measure is locally infinite on $J_{r}\left(F_{\lambda}\right)$ whereas the Hausdorff measure is on $J_{r}\left(F_{\lambda}\right)$ finite and positive. We also show that the Hausdorff dimension of $J_{r}\left(F_{\lambda}\right)$ is in the open interval $(1,2)$. All these geometric properties of the set $J_{r}\left(F_{\lambda}\right)$ clearly indicate that this is the right object to deal with. The inequality $\operatorname{HD}\left(J_{r}\left(F_{\lambda}\right)\right)>1$ follows from $\operatorname{HD}\left(J_{b d}\right)>1$. The inequality $\operatorname{HD}\left(J_{r}\left(F_{\lambda}\right)\right)<2$ is an immediate consequence of a modestly sounding fact that the $h_{\lambda}$-packing measure of $J_{r}\left(F_{\lambda}\right)$ is locally infinite. Several useful formulas used throughout the entire paper are placed in the appendix since they do not form the mainstream of arguments of our approach.

\section{Notation}

In this extremely short section we collect some not quite obvious notation used throughout the paper. For every $M \in \mathbb{R}$, let

$$
\mathbb{C}_{M}=\{z \in \mathbb{C}: \operatorname{Re} z<M\} \quad \text { and } \quad Q_{M}=\{z \in Q: \operatorname{Re} z<M\}
$$

For every $R>0$ let

$$
B_{-}(0, R):=\{z \in B(0, R): \operatorname{Re}(z)<0\}
$$


and if $0<r<R$, let

$$
A_{-}(0, r, R)=\{z \in \mathbb{C}: r<|z|<R, \operatorname{Re}(z)<0\} .
$$

From now onward we put

$$
f:=f_{\lambda} \quad \text { and } \quad F:=F_{\lambda} .
$$

\section{Bounded orbits}

In this section we will prove the fact which besides being interesting itself is absolutely necessary for the proofs of all further results of this paper. Let

$$
J_{b d}(F)=\left\{z \in J(F): \inf _{n \geq 1}\left\{\operatorname{Re}\left(F^{n}(z)\right)\right\}>-\infty \text { and } \inf _{n \geq 1}\left\{\left|F^{n}(z)\right|\right\}>0\right\} .
$$

For every $M>0$, let

$$
W_{M}=\{z \in J(F): \operatorname{Re}(z) \geq-M \text { and }|z| \geq 1 / M\}
$$

and let

$$
K_{M}=\bigcap_{k \geq 0} F^{-k}\left(W_{M}\right)
$$

The fact we just mentioned is this.

Lemma 3.1 We have $\operatorname{HD}\left(J_{b d}(F)\right)>1$. Even more, there exists $s>1$ such that $\operatorname{HD}\left(K_{M}\right) \geq$ s for all $M>0$ large enough.

Proof. Given $T_{4} \leq r_{1}<r_{2}$ it follows from (A.18) that

$$
f_{0}^{-1}\left(A_{-}\left(0, r_{1}, r_{2}\right)\right) \subset A\left(0, \frac{\lambda}{4 r_{2}}, \frac{\lambda}{r_{1}}\right) \text {. }
$$

The heart of our proof is to define and examine an appropriate iterated function system consisting of backward holomorphic branches of $F^{2}$. In order to do it we need some estimates. Fix $R>0$ to be determined in the course of the proof. For every $r \in(0, R)$ and every $k \geq 0$ put

$$
P_{k}(r, R)=\left\{z \in \mathbb{C}:-R<\operatorname{Re}(z)<-r, k \pi-\frac{\pi}{2}<\operatorname{Im}(z)<k \pi+\frac{\pi}{2}\right\}
$$

and notice that

$$
P_{k}(1, R) \subset A_{-}\left(0, \frac{1}{2}(1+k \pi), R+\left(k+\frac{1}{2}\right) \pi\right) \subset A_{-}\left(0, \frac{1}{2}(1+k \pi), 2 R+k \pi\right),
$$

where the latter inclusion was written assuming that $R \geq \pi / 2$. It then follows from (3.3) and (A.17) that for all $k \geq 1$ large enough, say $k \geq k_{0}$, we have

$$
\begin{aligned}
f_{0}^{-2}\left(P_{k}(1, R)\right) & \subset f_{0}^{-2}\left(A_{-}\left(0, \frac{1}{2}(1+k \pi), 2 R+k \pi\right)\right) \\
& \subset f_{0}^{-1}\left(A_{-}\left(0, \frac{\lambda}{4(2 R+k \pi)}, \frac{2 \lambda}{1+k \pi}\right)\right) \\
& \subset\left\{z \in P: c_{2}+\frac{1}{2} \log \left(\frac{\lambda}{4(2 R+k \pi)}\right)<\operatorname{Re} z<c_{1}+\frac{1}{2} \log \left(\frac{2 \lambda}{1+k \pi}\right)\right\} .
\end{aligned}
$$

Fix an integer $p \geq 2$ so large that $\frac{2 \lambda}{\pi} \exp \left(2\left(c_{1}+1+p j\right)\right) \geq k_{0}$, say $p \geq p_{0}$. Fix also an integer $q \geq 2$. If

$$
0 \leq k<\frac{\lambda}{4 \pi} \exp \left(2\left(c_{2}+1+p(j+1)\right)-\frac{2(1+p q)}{\pi},\right.
$$


then

$$
c_{2}+\frac{1}{2} \log \left(\frac{\lambda}{4(2(1+p q)+k \pi)}\right)>-1-p(j+1) .
$$

Also, if

$$
k>\frac{2 \lambda}{\pi} \exp \left(2\left(c_{1}+1+p j\right)\right)
$$

then

$$
c_{1}+\frac{1}{2} \log \left(\frac{2 \lambda}{1+k \pi}\right)<-1-p j .
$$

Utilizing therefore (3.4), we get for all $1 \leq j \leq q-1$ that

$$
f_{0}^{-2}\left(P_{k}(1,1+p q)\right) \subset P_{0}(1+p j, 1+p(j+1)) .
$$

For all integers $2 \leq q \leq \exp (3 p)$, where $p \geq p_{0}$ is large enough, we have

$$
\frac{\lambda}{8 \pi} \exp \left(2\left(c_{2}+1+p(j+1)\right)<\frac{\lambda}{4 \pi} \exp \left(2\left(c_{2}+1+p(j+1)\right)-\frac{2(1+p q)}{\pi} .\right.\right.
$$

For all $j=1, \ldots, q-1$ let $I_{j}:=\left[a_{j}, b_{j}\right] \cap \mathbb{N}$, where $a_{j}=\frac{2 \lambda}{\pi} \exp \left(2\left(c_{1}+1+p j\right)\right)=C_{4} e^{2 p j}$ and $b_{j}=$ $\frac{\lambda}{8 \pi} \exp \left(2\left(c_{2}+1+p(j+1)\right)=C_{5} e^{2 p} e^{2 p j}\right.$ with appropriate positive constants $C_{4}$ and $C_{5}$. Then

$$
\sharp\left(I_{j}\right)>\frac{1}{2} C_{5} e^{2 p} e^{2 p j} .
$$

For every $z \in P_{0}(1+p j, 1+p(j+1))$ it follows from (A.7) that $\left|\left(f^{2}\right)^{\prime}(z)\right| \leq C_{6} e^{2 p(j+1)}$ (with an appropriate positive constant $C_{6}$ ), and consequently

$$
\left|\left(f^{2}\right)^{\prime}(z)\right|^{-t} \geq C_{6}^{-t} e^{-2 p(j+1) t}
$$

Fixing $q=E\left(e^{3 p}\right)$ we now define the conformal iterated function system (see [21] for an account of the theory of conformal iterated function systems)

$$
\mathcal{S}_{p}=\left\{\phi_{j, k}: P_{0}(1,1+p q) \rightarrow P_{0}(1,1+p q)\right\}_{1 \leq j \leq q-1, k \in I_{j}},
$$

where $\phi_{j, k}(z)=f_{0}^{-2}(z+2 \pi i k)$. Fix $t \geq 0$ and let $\mathrm{P}(t)$ be the topological pressure corresponding to the parameter $t$ (see [21] for the definition of $\mathrm{P}(t)$ ). Since, by (3.5), for every $j \in\{1,2, \ldots, q-1\}$ we have

$$
\phi_{j, k}\left(P_{0}(1,1+p q)\right) \subset f_{0}^{-2}\left(P_{k}(1,1+p q)\right) \subset P_{0}(1+p j, 1+p(j+1)),
$$

it follows from (3.7) and (3.8) that

$$
\begin{aligned}
\mathrm{P}(t) & \geq \log \sum_{j=1}^{q-1} \sum_{k \in I_{j}} \inf \left\{\left|\left(F^{2}\right)^{\prime}(z)\right|^{-t}: z \in P_{0}(1+p j, 1+p(j+1))\right\} \\
& \geq \log \sum_{j=1}^{q-1} \sharp\left(I_{j}\right) C_{6}^{-t} e^{-2 p(j+1) t} \\
& \geq \log \left(\sum_{j=1}^{q-1} \frac{1}{2} C_{5} C_{6}^{-t} e^{2 p(j+1)} e^{-2 p(j+1) t}\right) \\
& \geq \log \sum_{j=1}^{q-1} e^{2 p(j+1)(1-t)}+C_{7}
\end{aligned}
$$


with an appropriate constant $C_{7} \in \mathbb{R}$ independent of $p$ and $q=E\left(e^{3 p}\right)$. Therefore

$$
\mathrm{P}(1) \geq \log (q-1)+C_{7}>\log \left(e^{3 p}-2\right)+C_{7}>0
$$

for all $p \geq 2$ large enough. Let $J_{p}$ be the limit set of the conformal iterated function system $\mathcal{S}_{p}$. It follows from [21, Theorem 3.15] that $\operatorname{HD}\left(J_{p}\right)>1$. Identifying the belt $P$ with $\Pi(P) \subset Q$, it follows from the construction of $S_{p}$ and the definition of the limit set $J_{p}$, that $F^{2}\left(J_{p}\right) \subset J_{p}$ and that $J_{p}$ is the closure of the fixed (attracting) points of all compositions of all maps from $\mathcal{S}_{p}$. Now, for every $z \in J_{p}$, the orbit $\left\{F^{2 n}(z)\right\}_{n \geq 0}$ is contained in $P_{0}(1,1+p q)$. Since in addition $\overline{J(F) \cap P_{0}(1,1+p q)}$ is a compact subset of $Q_{-1}$, we conclude that $J_{p} \subset K_{M}$ for all $M>0$ large enough. We are done.

\section{Conformal measures}

Our first goal in this section is to prove the existence of a conformal measure and to examine in detail its properties. In order to do it we will need to deal with the sets $K_{M}, M>0$, introduced in the previous section. Since $F: J(F) \rightarrow J(F)$ is continuous, $K_{M}$ is a forward $F$-invariant compact subset of $J(F)$. Notice also that if $z \in Q, j \geq 0, F^{j}(z) \in Q_{0}$ and $\left|F^{j}(z)\right| \geq 1 / M$, then there exists a unique holomorphic inverse branch $F_{z}^{-j}: B\left(F^{j}(z), 1 / M\right) \rightarrow Q$ of $F^{j}$, sending $F^{j}(z)$ to $z$. We shall prove the following.

Lemma 4.1 For all $M>0$ and every $\theta>1$ there exists $q_{M}(\theta) \geq 1$ such that $\left|\left(F^{k}\right)^{\prime}(x)\right| \geq \theta$ for all $x \in K_{M}$ and all $k \geq q_{M}(\theta)$.

Proof. Fix $M>0$ and suppose on the contrary that there exist a sequence $\left\{x_{i}\right\}_{i=1}^{\infty} \subset K_{M}$ and $\left\{n_{i}\right\}_{i=1}^{\infty}$, an unbounded increasing sequence of positive integers such that

$$
\left|\left(F^{n_{i}}\right)^{\prime}\left(x_{i}\right)\right|<\theta .
$$

Consider holomorphic inverse branches $F_{i}^{-n_{i}}: B\left(F^{n_{i}}\left(x_{i}\right), 1 / n\right) \rightarrow Q$ of $F^{n_{i}}$ sending $F^{n_{i}}\left(x_{i}\right)$ to $x_{i}$. Passing to a subsequence, we may assume that the limits $x:=\lim _{i \rightarrow \infty} x_{i} \in K_{M}$ and $y:=\lim _{i \rightarrow \infty} F^{n_{i}}\left(x_{i}\right) \in K_{M}$ exist. The formula (4.1) equivalently means that $\left|\left(F_{i}^{-n_{i}}\right)^{\prime}\left(F^{n_{i}}\left(x_{i}\right)\right)\right|>1 / \theta$ for all $i \geq 1$. Applying now the $\frac{1}{4}$-Koebe distortion theorem we see that

$$
F_{*}^{-n_{i}}\left(B\left(F^{n_{i}}\left(x_{i}\right), 1 / n\right)\right) \supset B\left(x_{i},(4 \theta n)^{-1}\right)
$$

for all $i \geq 1$. Therefore, $F_{*}^{-n_{i}}\left(B\left(F^{n_{i}}\left(x_{i}\right), 1 / n\right)\right) \supset B\left(x,(8 \theta n)^{-1}\right)$ for all $i \geq 1$ large enough. Hence $F^{n_{i}}\left(B\left(x,(8 \theta n)^{-1}\right)\right) \subset B\left(F^{n_{i}}\left(x_{i}\right), 1 / n\right) \subset B(y, 2 / n)$ for all $i \geq 1$ large enough. Thus the family

$$
\left\{\left.F^{n_{i}}\right|_{B\left(x,(8 \theta n)^{-1}\right)}\right\}_{i \geq 1}
$$

is normal. This however is a contradiction with the fact that $x_{i} \in K_{M} \subset J(F)$ and we are done.

Given $t \geq 0$ a Borel probability measure on $Q$ is said to be $t$-conformal for $F: J(F) \rightarrow J(F)$ if and only if $m(J(F))=1$ and

$$
m(F(A))=\int_{A}\left|F^{\prime}\right|^{t} d m
$$

for every Borel set $A \subset J(F)$ such that $\left.F\right|_{A}$ is one-to-one. First, following [10], for every $M>0$ large enough, we shall build a probability Borel measure $m_{M}$, with the topological support contained in $K_{M}$, and which will be "almost conformal" for some $t_{M} \geq 0$, meaning that

$$
m_{M}(F(A)) \geq \int_{A}\left|F^{\prime}\right|^{t_{M}} d m_{M}
$$

for every Borel set $A \subset Q$ such that $\left.F\right|_{A}$ is 1-to-1, and (4.2) holds if we assume in addition that $A \cap\{z \in Q$ : $\operatorname{Re} z \leq M$ or $|z| \leq 1 / M\}=\emptyset$. In what follows throughout Corollary 4.4 we follow closely the appropriate 
reasoning from [32]. In the sequel, we will need to refer to some details of the construction, of the measure $m_{M}$, so we briefly describe it now. For every $M>0$ large enough choose a finite set $E^{M} \subset K_{M}$ such that the $B\left(E^{M}, 1 / 2 M\right) \supset K_{M}$ and that $E^{M}$ contains the forward orbit of a periodic point $\xi$ of $F$. Notice that, since $K_{M}$ is $F$-forward invariant, the whole forward orbit of $\xi$ is contained in $K_{M}$. The existence of such a periodic point follows from the density of periodic points in $J(F)$. Consider the function

$$
c_{M}(t)=\limsup _{n \rightarrow \infty} \frac{1}{n} \log \sum_{x \in E_{M}} \sum_{w \in\left(\left.F\right|_{K_{M}}\right)^{-n}(x)}\left|\left(F^{n}\right)^{\prime}\right|^{-t}(w) .
$$

The function $t \mapsto c_{M}(t), t \in \mathbb{R}$, has three important properties. First, notice that it follows from Hölder's inequality that it is convex in $\mathbb{R}$, so it is continuous. Next, it follows easily from Lemma 4.1 that this function is strictly decreasing and $\lim _{t \rightarrow+\infty} c_{M}(t)=-\infty$. Finally, each set $\left(\left.F\right|_{K_{M}}\right)^{-n}\left(E^{M}\right)$ is not empty as it contains a point from the forward orbit of $\xi$. In particular $c_{M}(t) \geq 0$ for all $t \in \mathbb{R}$. All these properties imply that there exists a unique value $t=t_{M}$ with $c_{M}\left(t_{M}\right)=0$. Following the general construction described in [10] (see also [23, Chapter 10]), with the sets $E_{n}=\left(\left.F\right|_{K_{M}}\right)^{-n}\left(E^{M}\right)$ we obtain a measure $m_{M}$, for which $m_{M}\left(K_{M}\right)=1$ and which is "almost conformal" with the exponent $t_{M}$. We continue on with the following two lemmas; the idea of their proofs comes from [32].

Lemma 4.2 $\mathrm{HD}\left(K_{M}\right) \geq t_{M}$.

Proof. Fix a point $x \in K_{M}$ and an integer $n \geq 1$. Let $F_{x}^{-n}: B\left(F^{n}(x), 1 / M\right) \rightarrow Q$ be the holomorphic inverse branch of $F^{n}$ sending $F^{n}(x)$ to $x$. Applying now the $\frac{1}{4}$-Koebe distortion theorem and the standard Koebe distortion theorem, it follows from (4.3) that

$$
\begin{aligned}
m_{M}\left(B\left(x, \frac{1}{4}\left|\left(F^{n}\right)^{\prime}(x)\right|^{-1} \frac{1}{2 M}\right)\right) & \leq m_{M}\left(F_{x}^{-n}\left(B\left(F^{n}(x), \frac{1}{2 M}\right)\right)\right) \\
& \leq K^{t_{M}}\left|\left(F^{n}\right)^{\prime}(x)\right|^{-t_{M}} m_{M}\left(B\left(F^{n}(x), \frac{1}{2 M}\right)\right) \\
& \leq(8 K M)^{t_{M}}\left(\frac{1}{4}\left|\left(F^{n}\right)^{\prime}(x)\right|^{-1} \frac{1}{2 M}\right)^{t_{M}} .
\end{aligned}
$$

Since, by Lemma 4.1, $\lim _{n \rightarrow \infty}\left|\left(F^{n}\right)^{\prime}(x)\right|=\infty$ uniformly on $K_{M}$, we conclude that for every $r>0$ small enough there exists $n \geq 1$ such that

$$
\frac{1}{4}\left|\left(F^{n+1}\right)^{\prime}(x)\right|^{-1} \frac{1}{2 M} \leq r \leq \frac{1}{4}\left|\left(F^{n}\right)^{\prime}(x)\right|^{-1} \frac{1}{2 M} .
$$

Using this, (4.4) and the chain rule, we therefore get that

$$
m_{M}(B(x, r)) \leq\left((8 K T M)^{t_{M}}\right) r^{t_{M}},
$$

where $T=\sup \left\{\left|F^{\prime}(y)\right|: y \in K_{M}\right\}$ is finite since $K_{M}$ is a compact subset of $Q \backslash\{0\}$. This inequality implies in a standard way that $\operatorname{HD}\left(K_{M}\right) \geq t_{M}$ (see e.g. [23]).

Lemma 4.3 For every $M$ large enough there exists $p>0$ such that $\operatorname{HD}\left(K_{M}\right) \leq t_{M+p}$.

Proof. It easily follows from Lemma 4.1 and the absence of critical points of $F$ in $Q$ that

$$
L=\inf \left\{\left|\left(F^{n}\right)^{\prime}(w)\right|: w \in K_{M}, n \geq 1\right\}>0 \quad \text { and } \quad \lim _{n \rightarrow \infty}\left|\left(F^{n}\right)^{\prime}(z)\right|=\infty
$$

for all $z \in K_{M}$. Fix $p>0$ so large that $K L^{-1}<p(M(M+p))^{-1}$ and consider the set $J_{M+p}$. Following the construction of almost conformal measures described above, we choose a finite collection of points $E^{M+p} \subset$ $K_{M+p}$ such that the balls $B\left(x,(2(M+p))^{-1}\right), x \in E^{M+p}$, cover the set $K_{M+p}$. Let $y \in K_{M} \subset K_{M+p}$. Given $n \geq 0$ there exists $x \in E^{M+p}$ such that $F^{n}(y) \in B\left(x,(2(M+p))^{-1}\right)$. By our definition of the set $K_{M+p}$, all holomorphic branches of $F^{-i}, i \geq 0$, are well-defined on $B(x, 1 /(M+p))$. Fix $0 \leq i \leq n$ and let 
$F_{y}^{-i}$ be the holomorphic branch of $F^{-i}$ sending $F^{n}(y)$ to $F^{n-i}(y)$. Then, by Koebe's distortion theorem, for all $z \in B\left(x,(2(M+p))^{-1}\right)$, we get

$$
\frac{\left|\left(F_{y}^{-i}\right)^{\prime}(z)\right|}{\left|\left(F_{y}^{-i}\right)^{\prime}\left(F^{n}(y)\right)\right|} \leq K
$$

So, since $F^{n-i}(y) \in K_{M}$, using (4.5), we obtain $\left|\left(F_{y}^{-i}\right)^{\prime}(z)\right| \leq K\left|\left(F_{y}^{-i}\right)^{\prime}\left(F^{n}(y)\right)\right| \leq K L^{-1}$. Thus, $\left|F_{y}^{-i}(x)-F^{n-i}(y)\right| \leq K L^{-1}<p(M(M+p))^{-1}$, and consequently, using the fact that $F^{n-i}(y) \in W_{M}$, we see that $F_{y}^{-i}(x) \in W_{M+p}$ for all $0 \leq i \leq n$. This implies that $F_{y}^{-n}(x) \in K_{M+p}$, i.e., $F_{y}^{-n}(x) \in\left(\left.F\right|_{K_{M+p}}\right)^{-n}(x)$. Let $\mathcal{F}_{n}(x)$ be the collection of all holomorphic inverse branches $F_{\nu}^{-n}$ of $F^{n}$ defined on $B\left(x,(M+p)^{-1}\right)$, such that $F_{\nu}^{-n}(x) \in K_{M+p}$. It follows from the above considerations that

$$
K_{M} \subset \bigcup_{x \in E^{M+p}} \bigcup_{\nu \in \mathcal{F}_{n}(x)} F_{\nu}^{-n}\left(B\left(x,(2(M+p))^{-1}\right)\right)
$$

In addition, in view of Lemma 4.1, $\operatorname{diam}\left(F_{\nu}^{-n}\left(B\left(x,(2(M+p))^{-1}\right)\right)\right) \rightarrow 0$ uniformly as $n \rightarrow \infty$, and for every $t \geq 0$

$$
\sum_{x \in E^{M+p}} \sum_{\nu \in \mathcal{F}_{n}(x)}\left(\operatorname{diam}\left(F_{\nu}^{-n}\left(B\left(x, \delta_{M+p}\right)\right)\right)\right)^{t} \preceq \sum_{x \in E^{M+p}} \sum_{w \in\left(\left.F\right|_{K_{M+p}}\right)^{-n}(x)} \frac{1}{\left|\left(F^{n}\right)^{\prime}(w)\right|^{t}}
$$

Fix now an arbitrary $t>t_{M+p}$. Then $c_{M+p}(t)<0$ and

$$
\sum_{x \in E^{M+p}} \sum_{w \in\left(\left.F\right|_{K_{M+p}}\right)^{-n}(x)} \frac{1}{\left|\left(F^{n}\right)^{\prime}(w)\right|^{t}} \leq \exp \left(\frac{1}{2} c_{M+p}(t) n\right)
$$

for all $n$ large enough. Combining this, (4.6) and (4.7), we conclude that $\mathrm{H}_{t}\left(K_{M}\right)=0$ for all $t>t_{M+p}$ and, consequently, $\operatorname{HD}\left(K_{M}\right) \leq t_{M+p}$.

Corollary 4.4 There exists $s>1$ such that $t_{M} \geq s$ for all $M$ large enough.

Proof. In view of Lemma 3.1 $\mathrm{HD}\left(K_{M}\right)>1$ for all $M$ large enough. Fix one such $M$ and put $s=t_{M}$. Choose $p$ ascribed to this $M$ according to Lemma 4.3. Then, by this lemma, $t_{M+q} \geq \operatorname{HD}\left(K_{M}\right)=s>1$ for all $q \geq p$. We are done.

Suppose now that $r \in(0, R)$ with some $R>0$ sufficiently small and that $z, w \in\{\xi \in \mathbb{C}: r \leq|\xi| \leq e r\}$. It then follows from (A.3) that

$$
|\operatorname{Im}(f(z))-\operatorname{Im}(f(w))| \leq \frac{\lambda}{r} .
$$

We therefore get the following.

Lemma 4.5 If $r \in(0, R)$, then the map $F$ restricted to the annulus $\{z \in \mathbb{C}: r \leq|z| \leq$ er $\}$ is at most $\frac{\lambda}{\pi r}$-to-one.

Recall that a family $\mathcal{F}$ of Borel probability measures on a metric space $Y$ is called tight if for every $\epsilon>0$ there exist a compact set $Y_{\epsilon} \subset Y$ and a finite set $\mathcal{F}_{\epsilon} \subset \mathcal{F}$ such that $\nu\left(Y \backslash Y_{\epsilon}\right) \leq \epsilon$ for all $\nu \in \mathcal{F} \backslash \mathcal{F}_{\epsilon}$. The first step in the actual construction of a conformal measure (with an exponent $>1$ ) is provided by the following.

Lemma 4.6 The sequence $\left\{m_{n}\right\}_{n=1}^{\infty}$ is tight in $Q$.

Proof. For every $k \geq T_{4}$ put

$$
X_{k}=\{z \in J(F):-(k+1) \leq \operatorname{Re}(z)<-k\} \subset Q_{-T_{4}} .
$$


It follows from (A.5) that

$$
f\left(X_{k}\right) \subset A\left(0, \frac{\lambda}{2} e^{-2(k+1)}, 2 \lambda e^{-2 k}\right) .
$$

It therefore follows from Lemma 4.5 that there exists a universal constant $C>0$ such that $\left.F\right|_{f\left(X_{k}\right)}$ is at most $C e^{2 k}$-to-one. Hence, for every $n \geq 1$, so large that $2 \geq t_{n} \geq s>1$ ( $s$ given by Corollary 4.4), say $n \geq q$, we have using (A.7) that

$$
\begin{aligned}
1 \geq m_{n}\left(F^{2}\left(X_{k}\right)\right) & \geq\left(C e^{2 k}\right)^{-1} \int_{X_{k}}\left|\left(F^{2}\right)^{\prime}\right|^{t_{n}} d m_{n} \\
& \geq C^{-1} e^{-2 k} m_{n}\left(X_{k}\right)\left(\frac{e^{2 k}}{16}\right)^{t_{n}} \\
& \geq\left(2^{8} C\right)^{-1} e^{2 k\left(t_{n}-1\right)} m_{n}\left(X_{k}\right) \\
& \geq\left(2^{8} C\right)^{-1} e^{2 k(s-1)} m_{n}\left(X_{k}\right) .
\end{aligned}
$$

Hence $m_{n}\left(X_{k}\right) \leq 2{ }^{8} C e^{2 k(1-s)}$, and therefore for every $n \geq q$ and $M \geq 1$

$$
m_{n}\left(Q_{M}\right)=\sum_{k=M}^{\infty} m_{n}\left(X_{k}\right) \leq 2^{8} C \sum_{k=M}^{\infty} e^{2(1-s) k}=\frac{2^{8} C e^{2(1-s) M}}{1-e^{2(1-s)}} .
$$

Since the last expression in this formula converges to zero when $M \rightarrow \infty$, we therefore conclude that the sequence $\left\{m_{n}\right\}_{n=1}^{\infty}$ is tight.

Since $F\left(Q_{M}\right)$ is a punctured neighbourhood of 0 , the following lemma is a straightforward consequence of Lemma 4.6. However, we provide a simple direct proof, as one formula derived in the course of the proof will be used later.

Lemma 4.7 If $m$ is a weak accumulation point of the sequence $\left\{m_{n}\right\}_{n=1}^{\infty}$, then $m(\{0\})=0$.

Proof. The idea of this proof is the same as the idea of the proof of Lemma 4.6. For every $k \geq 0$ let

$$
R_{k}=\left\{z \in Q: R e^{-(k+1)} \leq|z| \leq R e^{-k}\right\} .
$$

Similarly to the proof of the previous lemma, we see from Lemma 4.5 that $\left.F\right|_{R_{k}}$ is at most $C e^{k}$ to one with some constant $C>0$ independent of $k$. Hence for all $n \geq q, q$ defined in the previous proof, using (A.4) and Lemma 4.5, we get that

$$
\begin{aligned}
1 \geq m_{n}\left(F\left(R_{k}\right)\right) & =\left(C e^{k}\right)^{-1} \int_{R_{k}}\left|F^{\prime}\right|^{t_{n}} d m_{n} \\
& \geq\left(C e^{k}\right)^{-1}(\lambda / 4)^{t_{n}}\left(R^{-2} e^{2 k}\right)^{t_{n}} m_{n}\left(R_{k}\right) \\
& \succeq e^{-k} e^{2 k s} m_{n}\left(R_{k}\right) \\
& =e^{(2 s-1) k} m_{n}\left(R_{k}\right) .
\end{aligned}
$$

Hence

$$
m_{n}\left(R_{k}\right) \preceq e^{(1-2 s) k},
$$

and therefore, for every $n \geq q$ and every $j \geq 0$ we get

$$
m_{n}\left(B\left(0, R e^{-j}\right)\right)=\sum_{k=j}^{\infty} m_{n}\left(R_{k}\right) \preceq \sum_{k=j}^{\infty} e^{(1-2 s) k}=\frac{e^{(1-2 s) j}}{1-e^{(1-2 s)}} .
$$

Letting now $j \rightarrow \infty$, we conclude that $m(0)=0$. 
Since, in view of Lemma 3.1, $t_{n} \in[s, 2]$, with $s>1$, for all $n \geq 1$ large enough, we can choose a subsequence $\left\{n_{k}\right\}_{k=1}^{\infty}$ such that $\left\{t_{n_{k}}\right\}_{k=1}^{\infty}$ converges. Denote its limit by $h \in[s, 2]$. It follows from Lemma 4.6 and Prohorov's theorem (see [6, Theorem 5.1, Section 5, p. 59]) that passing yet to another subsequence, we may assume that the sequence $\left\{m_{n_{k}}\right\}_{k=1}^{\infty}$ converges weakly, say to a Borel probability measure $m$. Since all the measures $m_{n_{k}}$, $k \geq 1$, are supported on $J(F)$, so is $m$. Since there can be a problem with conformality of measures $m_{n_{k}}$ only on sets $\left\{z \in J(F): \operatorname{Re}(z)=-n_{k}\right.$ or $\left.|z|=1 / n\right\}$, since $\lim _{k \rightarrow \infty} n_{k}=\infty$, and since $F: J(F) \rightarrow J(F)$ is open and has no singular points except for 0 (which, because of Lemma 4.7, does not matter), proceeding with obvious modifications as in [10] (comp. [23]) and using Lemma 4.7 we obtain the following.

Theorem 4.8 The weak limit measure $m$ (at the moment depending on the sequence $\left\{n_{k}\right\}_{k=1}^{\infty}$ but see Theorem 4.13 for its uniqueness) is $h$-conformal for $F: J(F) \rightarrow J(F)$ with some $h \in(1,2]$ and $m(0)=0$.

Since, for every nonempty open set $U \subset J(F), \bigcup_{n \geq 0} F^{n}(U) \supset J(F) \backslash\{0\}$, we get the following immediate consequence of Theorem 4.8 .

Proposition 4.9 The h-conformal measure $m$ is positive on nonempty open subsets of $J(F)$.

For every $z \in Q$ let $\omega(z) \subset Q \cup\{-\infty\}$ be the $\omega$-limit set of the point $z$ with respect to the dynamical system $F: Q \rightarrow Q \cup\{-\infty\}$. We recall now the definition of the main object of our interest in this paper.

$$
J_{r}(F):=\{z \in Q: \omega(z) \backslash\{0,-\infty\} \neq \emptyset\}=\{z \in Q: \omega(z) \not \subset\{0,-\infty\} .
$$

We need one related definition. Let for $T>0$

$$
J_{r, T}(F)=\left\{z \in Q: \omega(z) \text { is not contained in } W_{T}^{c}\right\},
$$

where, we recall,

$$
W_{T}=\{z \in J(F): \operatorname{Re}(z) \geq-T \text { and }|z| \geq 1 / T\}
$$

Obviously

$$
J_{r}(F)=\bigcup_{n \geq 1} J_{r, n}(F) .
$$

Theorem 4.10 There exists $M>0$ such that if $\nu$ is $t$-conformal measure for $F: J(F) \rightarrow J(F)$ with $t>1$, then $\nu\left(J_{r, M}(F)\right)=1$. In particular $\nu\left(J_{r}(F)\right)=1$.

Proof. We shall prove first that there exists an integer $T_{5} \geq 1$ so large that

$$
\nu(F(A)) \geq 4 \nu(A)
$$

for every Borel set $A \subset B\left(0, R e^{-T_{5}}\right) \cap Q$. And indeed, suppose for a contrary that for every integer $k \geq 1$ there exists a Borel set $A_{k} \subset B\left(0, R e^{-k}\right)$ such that $\nu\left(F\left(A_{k}\right)\right)<4 \nu\left(A_{k}\right)$. Proceeding in the same way as in Lemma 4.7, we get for every $j \geq k$ the following.

$$
\begin{aligned}
4 \nu\left(A_{k}\right)>\nu\left(F\left(A_{k}\right)\right) & \geq \nu\left(F\left(A_{k} \cap R_{j}\right)\right) \\
& \geq\left(C e^{j}\right)^{-1} \int_{A_{k} \cap R_{j}}\left|F^{\prime}\right|^{t} d \nu \\
& \geq C^{-1} e^{-j}(\lambda / 4)^{t}\left(R^{-2} e^{2 j}\right)^{t} \nu\left(A_{k} \cap R_{j}\right) \\
& \geq C_{1} e^{j(2 t-1)} \nu\left(A_{k} \cap R_{j}\right)
\end{aligned}
$$

with some universal constant $C_{1}>0$. Hence, $\nu\left(A_{k} \cap R_{j}\right) \leq 4 C_{1}^{-1} e^{j(1-2 t)} \nu\left(A_{k}\right)$. Therefore, summing over all $j \geq k$, we get

$$
\nu\left(A_{k}\right)=\sum_{j=k}^{\infty} \nu\left(A_{k} \cap R_{j}\right) \leq 4 C_{1}^{-1} \nu\left(A_{k}\right) \sum_{j=k}^{\infty} e^{j(1-2 t)}=\frac{4 e^{k(1-2 t)}}{C_{1}\left(1-e^{(1-2 t)}\right)} \nu\left(A_{k}\right) .
$$


Since $\nu\left(A_{k}\right)>0$, we therefore get

$$
1 \leq \frac{4 e^{(1-2 t) k}}{C_{1}\left(1-e^{(1-2 t)}\right)} .
$$

Since the right-hand side of this inequality converges to 0 when $k \rightarrow \infty$, we get a contradiction, and formula (4.10) is proven. Proceeding now similarly as above and similarly as in the proof of Lemma 4.6 we shall demonstrate that there exists an integer $T_{6}$ so large that

$$
\nu\left(F^{2}(A)\right) \geq 4 \nu(A)
$$

for every Borel set $A \subset Q_{-T_{6}}$. And indeed, suppose for a contrary that for every $k \geq T_{6}$ there exists a Borel set $E_{k} \subset Q_{-k}$ such that $\nu\left(F^{2}\left(E_{k}\right)\right)<4 \nu\left(E_{k}\right)$. Proceeding in the same way as in Lemma 4.6, we get for every $j \geq k$ that

$$
\begin{aligned}
4 \nu\left(E_{k}\right)>\nu\left(F^{2}\left(E_{k}\right)\right) & \geq \nu\left(F^{2}\left(E_{k} \cap X_{j}\right)\right) \\
& \geq\left(C e^{2 j}\right)^{-1} \int_{E_{k} \cap X_{j}}\left|\left(F^{2}\right)^{\prime}\right|^{t} d \nu \\
& \geq C^{-1} e^{-2 j}\left(\frac{1}{16} e^{2 j}\right)^{t} \nu\left(E_{k} \cap X_{j}\right) \\
& \geq C^{-1} e^{2 j(t-1)} \nu\left(E_{k} \cap X_{j}\right)
\end{aligned}
$$

The rest of the argument is exactly the same as the corresponding part of the proof of (4.10). Now set

$$
T_{7}=\max \left\{T_{5}, T_{6}\right\}
$$

and for every $k \geq T_{7}$ put

$$
\begin{aligned}
I_{k}=I_{k}(F) & =\bigcap_{n \geq 0} F^{-n}\left(W_{k}^{c}\right) \\
& =\left\{z \in J(F): \operatorname{Re}\left(F^{n}(z)\right)<-k \text { or }\left|F^{n}(z)\right|<1 / k \text { for all } n \geq 0\right\} .
\end{aligned}
$$

Note that $F\left(I_{k}\right) \subset I_{k}$. We shall prove that there is $M>0$ so large that for all $k \geq M, \nu\left(I_{k}\right)=0$. Indeed, set

$$
I_{k}^{\infty}:=I_{k} \cap Q_{-k}, \quad I_{k}^{0}:=I_{k} \cap B(0,1 / k) .
$$

Then

$$
I_{k}=I_{k}^{\infty} \cup I_{k}^{0}
$$

For every $A \subset I_{k}$ define

$$
G(A):=F^{2}\left(A \cap I_{k}^{\infty}\right) \cup F\left(A \cap I_{k}^{0}\right) .
$$

Applying (4.10) and (4.11), we get for all $k \geq 1$ large enough that

$$
\begin{aligned}
\nu(G(A)) & \geq \frac{1}{2}\left(\nu\left(F^{2}\left(A \cap I_{k}^{\infty}\right)\right)+\nu\left(F\left(A \cap I_{k}^{0}\right)\right)\right) \\
& \geq \frac{1}{2} 4 \nu\left(A \cap I_{k}^{\infty}\right)+4 \nu\left(A \cap I_{k}^{0}\right) \\
& =2 \nu(A) .
\end{aligned}
$$

Since $A \subset I_{k}$ and $F\left(I_{k}\right) \subset I_{k}$ (so also $F^{2}\left(I_{k}\right) \subset I_{k}$ ), it follows from (4.12) that $G(A) \subset I_{k}$. Hence $G^{n}(A) \subset$ $I_{k}$ for all $n \geq 1$. We apply now (4.13) to the set $A=I_{k}$ and obtain

$$
\nu\left(G^{n}\left(I_{k}\right)\right) \geq 2^{n} \nu\left(I_{k}\right)
$$


for all $n \geq 1$. Since $\nu$ is a probability measure, $\nu\left(I_{k}\right)=0$ for all $k \geq 1$ large enough. In particular $\nu\left(I_{M}\right)=0$, and consequently

$$
\nu\left(\bigcup_{j=0}^{\infty} F^{-j}\left(I_{M}\right)\right)=0
$$

Since

$$
J_{r}(F) \backslash J_{r, M}(F) \subset\left\{z \in \mathbb{C}: \exists k \geq 0 F^{k}(z) \in I_{M}\right\}=\bigcup_{k \geq 0} F^{-k}\left(I_{M}\right)
$$

applying (4.14), we get $\nu\left(J_{r}(F) \backslash J_{r, M}(F)\right)=0$. Hence $\nu\left(J_{r, M}(F)\right)=1$ and we are done.

Now fix $T>0$. It follows from the definition of the set $J_{r, T}(F)$ that there exists an unbounded increasing sequence $\left\{n_{k}(z)\right\}_{k=1}^{\infty}$ such that $y(z):=\lim _{k \rightarrow \infty} F^{n_{k}(z)}(z)$ exists and

$$
F^{n_{k}(z)}(z) \in Q_{-2 T}^{c} \backslash B\left(0, \frac{1}{2 T}\right)
$$

for all $k \geq 1$. We shall prove the following.

Lemma 4.11 For every $T \geq 1$ and every $z \in J_{r, T}(F)$

$$
\lim _{k \rightarrow \infty}\left|\left(F^{n_{k}(z)}\right)^{\prime}(z)\right|=+\infty .
$$

Proof. Put $n_{k}=n_{k}(z), z \in J_{r, T}(F), k \geq 1$. Suppose on the contrary that

$$
\liminf _{k \rightarrow \infty}\left|\left(F^{n_{k}}\right)^{\prime}(z)\right|<+\infty \text {. }
$$

Without loss of generality we may assume that $\tau:=\lim _{k \rightarrow \infty}\left|\left(F^{n_{k}}\right)^{\prime}(z)\right|<+\infty$ and that $F^{n_{k}}(z) \in$ $B\left(y(z),(6 T)^{-1}\right)$ for all $k \geq 1$. Consider the family $\left\{F_{z}^{-n_{k}}: B\left(y(z),(2 T)^{-1}\right) \rightarrow Q\right\}_{k \geq 1}$ of holomorphic inverse branches of $F^{n_{k}}$ sending $F^{n_{k}}(z)$ to $z$. Applying the $\frac{1}{4}$-Koebe's distortion theorem, we see that $F_{z}^{-n_{k}}\left(B\left(y(z),(2 T)^{-1}\right)\right) \supset B\left(z,(8 \tau)^{-1}\right)$ for all $k \geq 1$ large enough. Thus $F^{n_{k}}\left(B\left(z,(8 \tau)^{-1}\right)\right) \subset$ $B\left(y(z),(2 T)^{-1}\right)$, and consequently the family of maps $\left\{F^{n_{k}}: B\left(z,(8 \tau)^{-1}\right) \rightarrow Q\right\}_{k=1}^{\infty}$ is normal, which contradicts the fact that $z \in J(F)$ and finishes the proof.

Lemma 4.12 Let $m$ be the weak-limit measure whose h-conformality is established in Theorem 4.8. Then for every nonempty open set $U \subset Q$, we have

$$
\limsup _{n \rightarrow \infty} m\left(F^{n}(U)\right)=1 .
$$

Proof. Let $\widetilde{U}$ be a connected component of $\Pi^{-1}(U)$. Since periodic points of $f$ are dense in $J(f), \widetilde{U}$ contains a repelling periodic point $\omega$. Denote the period of $\omega$ by $p$. There then exists an open ball $W \subset \widetilde{U}$ centered at $\omega$ such that $f^{p}(W) \supset W$. Since $\omega \in J\left(f^{p}\right), \bigcup_{n \geq 0} f^{p n}(W)=\mathbb{C} \backslash\{0, \lambda\}$. Hence for every $n \geq 1$

and

$$
F^{p n}(\Pi(W))=\Pi\left(f^{p n}(W)\right) \supset \Pi(W)
$$

$$
\bigcup_{n \geq 0} F^{p n}(\Pi(W))=\bigcup_{n \geq 0} \Pi\left(f^{p n}(W)\right)=\Pi\left(\bigcup_{n \geq 0} f^{p n}(W)\right)=\Pi(\mathbb{C} \backslash\{0, \lambda\})=Q .
$$

Thus

$$
\lim _{n \rightarrow \infty} m\left(F^{p n}(\Pi(W))\right)=m\left(\bigcup_{n \geq 0} F^{p n}(\Pi(W))\right)=m(Q)=1 .
$$

Since $\Pi(W) \subset \Pi(\widetilde{U})=U$, we are done. 
The next theorem is the (important) starting point for the developing the ergodic theory of the dynamical system $F: J(F) \rightarrow J(F)$ with respect to the $h$-conformal measure $m$. The first elements of its proof go back to [26], the method matured in [27] and [28]. Its versions also can be for example found in [8], [19], [29], [31], and [32]. The full proof of Theorem 4.13 can be carried out with obvious minor modifications similarly to the proofs of [19, Theorem 4.23] and [31, Theorem 3.15].

Theorem 4.13 The h-conformal measure $m$ is a unique $t$-conformal probability measure, with $t>1$, for $F: J(F) \rightarrow J(F)$. In addition, $m$ is conservative and ergodic.

Combining together Theorems 4.8, 4.10, and 4.13, we can compactly collect the main results of this section in the following single theorem.

Theorem 4.14 The Hausdorff dimension $h$ is a unique exponent $t>1$ for which a $t$-conformal measure exists. There exists a unique $h$-conformal measure, which from now on will be denoted by $m$. The measure $m$ is ergodic, conservative and $m\left(J_{r, M}(F)\right)=1$.

\section{An invariant measure equivalent to the conformal measure $m$}

In this section we show the existence and uniqueness of a Borel probability $F$-invariant measure equivalent to $m$. We first prove the following.

Lemma 5.1 Up to a multiplicative constant there exists a unique F-invariant, $\sigma$-finite measure $\mu$, which is conservative, ergodic and equivalent to the $h$-conformal measure $m$.

The idea of the proof of Lemma 5.1 is to apply a general sufficient condition for the existence of $\sigma$-finite absolutely continuous invariant measure proven in [20]. In order to formulate this condition suppose that $X$ is a $\sigma$-compact metric space, $m$ is a Borel probability measure on $X$, positive on open sets, and that a measurable map $T: X \rightarrow X$ is given with respect to which measure $m$ is quasi-invariant, i.e., $m \circ T^{-1}<<m$. Moreover we assume the existence of a countable partition $\alpha=\left\{A_{n}: n \geq 0\right\}$ of subsets of $X$ which are all $\sigma$-compact and of positive measure $m$. We also assume that $m\left(X \backslash \bigcup_{n \geq 0} A_{n}\right)=0$, and if additionally for all $m, n \geq 1$ there exists $k \geq 0$ such that

$$
m\left(T^{-k}\left(A_{m}\right) \cap A_{n}\right)>0,
$$

then the partition $\alpha$ is called irreducible. Martens' result comprising [20, Proposition 2.6 and Theorem 2.9] says the following.

Theorem 5.2 Suppose that $\alpha=\left\{A_{n}: n \geq 0\right\}$ is an irreducible partition for $T: X \rightarrow X$. Suppose that $T$ is conservative and ergodic with respect to the measure $m$. If for every $n \geq 1$ there exists $K_{n} \geq 1$ such that for all $k \geq 0$ and all Borel subsets $A$ of $A_{n}$

$$
K_{n}^{-1} \frac{m(A)}{m\left(A_{n}\right)} \leq \frac{m\left(T^{-k}(A)\right)}{m\left(T^{-k}\left(A_{n}\right)\right)} \leq K_{n} \frac{m(A)}{m\left(A_{n}\right)},
$$

then $T$ has a $\sigma$-finite $T$-invariant measure $\mu$ absolutely continuous with respect to $m$. Additionally $\mu$ is equivalent with $m$, conservative and ergodic, and unique up to a multiplicative constant.

Notice that the tempting trivial choice of $\alpha$ consisting of $A_{0}=X$ and $A_{n}=\emptyset$ for all $n \geq 1$ may make (5.2) fail to hold. If however (5.2) is true for all Borel subsets $A$ of $X$ (and $A_{0}=X$ ), then the resulting measure $\mu$ is finite.

Proof of Lemma 5.1 (sketch). Since in the sequel we will not only need Lemma 5.1 but a bit more, namely the way in which the invariant measure claimed in Theorem 5.2 is produced, we shall also describe this procedure briefly. Following Martens, one considers the following sequences of measures

$$
S_{k} m=\sum_{i=0}^{k-1} m \circ T^{-i} \quad \text { and } \quad Q_{k} m=\frac{S_{k} m}{S_{k} m\left(A_{0}\right)} .
$$


It is proven in [20] that each weak limit $\mu$ of the sequence $Q_{k}(m)$ has the properties required in Theorem 5.2, where a sequence $\left\{\nu_{k}: k \geq 1\right\}$ of measures on $X$ is said to converge weakly if for all $n \geq 1$ the measures $\nu_{k}$ converge weakly on all compact subsets of $A_{n}$. In fact it turns out that the sequence $Q_{k} m$ converges and

$$
\mu(F)=\lim _{n \rightarrow \infty} Q_{k} m(F)
$$

for every Borel set $F \subset X$. Making use of (5.1) and (5.2) one proves (see [20, Lemma 2.4]) the following.

Lemma 5.3 For every $n \geq 0$ we have $0<\mu\left(A_{n}\right)<\infty$. Furthermore, the Radon-Nikodym derivative $\frac{d \mu}{d m}$ is bounded above and below on $A_{n}$.

Let us pass now to our map $F: Q \backslash\{0\} \rightarrow Q$. The ergodicity and conservativity of the measure $m$ is proven in Theorem 4.13. Thus, we only need to construct an irreducible partition $\alpha$ with property (5.2). Indeed, set $Y=J(F) \backslash\{0\}$ and for every $y \in Y$ consider a ball $B(y, r(y)) \subset Q$ such that $r(y)>0, m(\partial B(y, r(y)))=0$, and $r(y)<(1 / 2) \min \{\pi / 2$, dist $(y, 0)\}$. The balls $B(y, r(y)), y \in Y$, cover $Y$ and since $Y$ is a metric separable space, one can choose a countable cover, say $\left\{\tilde{A}_{n}: n \geq 0\right\}$, from them. We may additionally require that the family $\left\{\tilde{A}_{n}: n \geq 0\right\}$ is locally finite that is that each point $x \in Y$ has an open neighborhood intersecting only finitely many balls $\tilde{A}_{n}, n \geq 0$. We now define the family $\alpha=\left\{A_{n}: n \geq 0\right\}$ inductively by setting

$$
A_{0}=\tilde{A}_{0} \quad \text { and } \quad A_{n+1}=\tilde{A}_{n+1} \backslash \bigcup_{k=1}^{n} \overline{\tilde{A}_{n}}
$$

(and throwing away empty sets). Obviously $\alpha$ is a disjoint family and

$$
\bigcup_{n \geq 1} A_{n} \supset J(F) \backslash\{0\} \backslash \bigcup_{n \geq 0} \partial \tilde{A}_{n} .
$$

Hence $m\left(\bigcup_{n \geq 0} A_{n}\right)=1$. The distortion condition (5.2) follows now from Koebe's distortion theorem with all

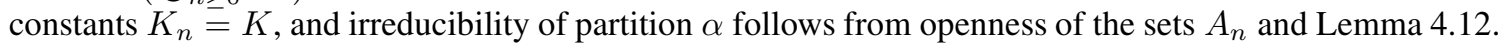

Now we are ready to prove the main theorem of this section.

Theorem 5.4 The $\sigma$-finite $F$-invariant measure $\mu$ equivalent to the $h$-conformal measure $m$ proven to exists in Lemma 5.1 is finite.

Proof. It suffices to show that there is $M>0$ such that $\mu\left(Q_{-M}\right)<\infty$ and $\mu\left(B\left(0, R e^{-M}\right)\right)<\infty$. Let us start with the inequality $\mu\left(Q_{-M}\right)<\infty$. For every $k \geq T_{4}$ the sets

$$
X_{k}=\{z \in J(F):-(k+1) \leq \operatorname{Re}(z)<-k\} \subset \mathbb{C}_{-T_{4}}
$$

have the same meaning as in the proof of Lemma 4.6. Exactly the same argument as that used in the proof of Lemma 4.6 shows that

$$
m\left(X_{k}\right) \leq 2^{8} C e^{2 k(1-h)} .
$$

We may assume without loss of generality that $A_{0}=X_{T_{4}}$. Fix $k \geq T_{4}$. Let

$$
S_{k}=[-(k+2),-2] \times[-k / 2, k / 2] \subset \mathbb{C}_{0} .
$$

The set $\{z \in \mathbb{C}: \operatorname{Im} z=(\pi / 2)\}$ is canonically embedded into $\mathbb{C}$, and therefore each holomorphic inverse branch $F_{*}^{-j}: Q \backslash \Pi(\{z \in \mathbb{C}: \operatorname{Im} z=(\pi / 2)\}) \rightarrow Q$ of $F^{j}, j \geq 1$, can be treated as defined on a subset of the complex plane $\mathbb{C}$. This map restricted to $X_{k}$ extends in a holomorphically univalent fashion to the set $S_{k}$. It therefore follows from Koebe's distortion theorem that there exists a constant $C_{1}>0$ such that for every $j \geq 1$, every $x \in X_{T_{3}}=A_{0}$ and every $y \in X_{k}$, we have

$$
\frac{\left|\left(F_{*}^{-j}\right)^{\prime}(y)\right|}{\left|\left(F_{*}^{-j}\right)^{\prime}(x)\right|} \leq C_{1} k^{3} \text {. }
$$


Therefore, using in addition Lemma 5.4, we obtain

$$
\frac{m\left(F_{*}^{-j}\left(X_{k}\right)\right)}{m\left(F_{*}^{-j}\left(A_{0}\right)\right)} \leq C_{1}^{h} k^{3 h} \frac{m\left(X_{k}\right)}{m\left(A_{0}\right)} \leq 2^{8} C_{1}^{h} k^{3 h} C m\left(A_{0}\right)^{-1} e^{2(1-h) k} .
$$

Hence

$$
\frac{m\left(F^{-j}\left(X_{k}\right)\right)}{m\left(F^{-j}\left(A_{0}\right)\right)} \leq 2^{8} C C_{1}^{h} m\left(A_{0}\right)^{-1} k^{3 h} e^{2(1-h) k}
$$

and consequently, for every $n \geq 0$,

$$
\frac{\sum_{j=0}^{n} m\left(F^{-j}\left(X_{k}\right)\right)}{\sum_{j=0}^{k} m\left(F^{-j}\left(A_{0}\right)\right)} \leq 2^{8} C C_{1}^{h} m\left(A_{0}\right)^{-1} k^{3 h} e^{2(1-h) k} .
$$

Thus, applying Theorem 5.2 we get

$$
\mu\left(X_{k}\right)=\lim _{k \rightarrow \infty} \frac{\sum_{j=0}^{k} m\left(F^{-j}\left(X_{k}\right)\right)}{\sum_{j=0}^{k} m\left(F^{-j}\left(A_{0}\right)\right)} \leq 2^{8} C C_{1}^{h} m\left(A_{0}\right)^{-1} k^{3 h} e^{2(1-h) k} .
$$

Hence

$$
\mu\left(Q_{-T_{3}}\right) \leq \sum_{k=T_{3}}^{\infty} \mu\left(X_{k}\right) \leq \sum_{k=T_{3}}^{\infty} 2^{8} C C_{1}^{h} m\left(A_{0}\right)^{-1} k^{3 h} e^{2(1-h) k}<\infty
$$

Let us now prove that $\mu\left(B\left(0, R e^{-M}\right)\right)<\infty$ for some $M$ large enough. We may assume without loss of generality that $A_{0}=R_{M}$, where $M$ will be determined later in the course of the proof and $R_{M}$ was defined at the beginning of the proof of Lemma 4.7. Fix $j \geq 0$, assume that $M$ is large enough, and for all $l \in \mathbb{Z} \backslash\{0\}$ consider all the holomorphic inverse branches $F_{*}^{-j}: B_{-}\left(0, R e^{-M}\right) \rightarrow Q$ of $F^{j}$ such that $f^{j}\left(F_{*}^{-j}\left(B\left(0, R e^{-M}\right)\right)=\right.$ $B\left(\pi l i, R e^{-M}\right)$ or equivalently $f^{j} \circ F_{*}^{-j}(z)=z+\pi i l$, which slightly informally means that constructing $F_{*}^{-j}$ we first translate the ball $B\left(\pi l i, R e^{-M}\right)$ (treated as a subset of $\mathbb{C}$ ) by the vector $\pi i l$, which brings us far away $(l \neq 0)$ from the singularity zero, and then we take any inverse branch of $f^{j}$ composed at the last step with the canonical projection $\Pi$. The Koebe's distortion theorem for all those inverse branches $F_{*}^{-j}$ applies and we get for every $k \geq M$ that

$$
\frac{m\left(F_{*}^{-j}\left(R_{k}\right)\right)}{m\left(F_{*}^{-j}\left(A_{0}\right)\right)} \leq K^{h} \frac{m\left(R_{k}\right)}{m\left(A_{0}\right)} .
$$

Recall that $K \geq 1$ comes from Koebe's distortion theorem. In exactly the same way as (4.8) we get the following

$$
m\left(R_{k}\right) \leq L e^{(1-2 h) k}
$$

for some constant $L>0$ and all $k \geq 0$. Combining this with (5.5), we obtain

$$
\frac{m\left(F_{*}^{-j}\left(R_{k}\right)\right)}{m\left(F_{*}^{-j}\left(A_{0}\right)\right)} \leq \frac{L K^{h}}{m\left(A_{0}\right)} e^{(1-2 h) k}
$$

Let now $F_{0}^{-j}: B_{-}(0, R) \rightarrow Q$ be a holomorphic branch of $F^{j}$ such that, abusing slightly notation, $\left.f^{j} \circ F_{0}^{-j}\left(R_{k}\right)\right)=R_{k}$. It follows from (A.10) (applied for $f_{0}^{-1}$ ) that

$$
F^{j-1}\left(F_{0}^{-j}\left(\bigcup_{s=M}^{k} R_{s}\right)\right) \subset\left\{z \in Q: \zeta-\frac{1}{2}(k+1) \leq \operatorname{Re}(z) \leq-2\right\}
$$


with some $\zeta>0$ and every $M$ large enough. Now, the same argument as used in the first part of this proof gives that

$$
\frac{m\left(F_{0}^{-j}\left(R_{k}\right)\right)}{m\left(F_{0}^{-j}\left(A_{0}\right)\right)} \leq C_{1} k^{3 h} \frac{m\left(F^{j-1}\left(F_{0}^{-j}\left(R_{k}\right)\right)\right)}{m\left(F^{j-1}\left(F_{0}^{-j}\left(A_{0}\right)\right)\right)}
$$

with some constant $C_{1}>0$. Using now (5.6) and (A.12) we conclude that with $M$ large enough

$$
\frac{m\left(F_{0}^{-j}\left(R_{k}\right)\right)}{m\left(F_{0}^{-j}\left(A_{0}\right)\right)} \leq C_{2} k^{3 h} \frac{e^{k h} m\left(R_{k}\right)}{e^{M h} m\left(A_{0}\right)}=\frac{C_{2}}{e^{M h} m\left(A_{0}\right)} k^{3 h} e^{k h} m\left(R_{k}\right) \leq \frac{L C_{2}}{e^{M h} m\left(A_{0}\right)} k^{3 h} e^{(1-h) k} .
$$

Combining this with (5.7), we see that for every $j \geq 1$ and every $k \geq M$

$$
\frac{m\left(F^{-j}\left(R_{k}\right)\right)}{m\left(F^{-j}\left(A_{0}\right)\right)} \leq C_{3} k^{3 h} e^{(1-h) k}
$$

with some universal constant $C_{3}>0$. Summing up over all $k \geq M$, we get

$$
\frac{m\left(F^{-j}\left(B\left(0, R e^{-M}\right)\right)\right)}{m\left(F^{-j}\left(A_{0}\right)\right)} \leq C_{3} \sum_{k=M}^{\infty} k^{3 h} e^{(1-h) k}<\infty
$$

Thus, for every $n \geq 0$

$$
\frac{\sum_{j=0}^{n} m\left(F^{-j}\left(B\left(0, R e^{-M}\right)\right)\right)}{\sum_{j=0}^{n} m\left(F^{-j}\left(A_{0}\right)\right)} \leq C_{3} \sum_{k=M}^{\infty} k^{3 h} e^{(1-h) k} .
$$

Hence, applying Theorem 5.2 and the construction of the measure $\mu$ given by (5.3), we conclude that

$$
\mu\left(B\left(0, R e^{-M}\right)\right) \leq C_{3} \sum_{k=M}^{\infty} k^{3 h} e^{(1-h) k}<\infty .
$$

We are done.

As an immediate consequence of this theorem and Lemma 5.1, we get the following.

Corollary 5.5 There exists a unique Borel probability F-invariant measure $\mu$ absolutely continuous with respect to the $h$-conformal measure $m$. In addition the invariant measure $\mu$ is ergodic and equivalent to $m$.

\section{Hausdorff and packing measures and dimensions}

Let $\mathrm{H}^{h}$ and $\mathrm{P}^{h}$ be respectively the $h$-dimensional Hausdorff and packing measures (see [25], comp. [23] for example, for its definition and some basic properties). The results of this section provide in a sense a complete description of the geometrical structure of the sets $J_{r}(F)$ and $J_{r}(f)$ and also they exhibit the geometrical meaning of the $h$-conformal measure $m$. The short proof of the first result improves on the argument from the proof of [29, Proposition 4.9].

Proposition 6.1 We have $\mathrm{P}^{h}\left(J_{r}(f)\right)=\mathrm{P}^{h}\left(J_{r}(F)\right)=\infty$. In fact $\mathrm{P}^{h}(G)=\infty$ for everyopen nonempty subset G of $J_{r}(f)$.

Proof. Since $m\left(J_{r}(F) \cap Q_{M}\right)>0$ for every $M \in \mathbb{R}$, it follows from ergodicity and conservativity of the measure $m$ (see Theorem 4.13) that there exists a set $E \subset J_{r}(F)$ such that $m(E)=1$ and $\liminf _{n \rightarrow \infty} \operatorname{Re} F^{n}(z)=$ $-\infty$ for every $z \in E$. Fix $z \in E$. This formula means that there exists an unbounded increasing sequence $\left\{n_{k}\right\}_{k=1}^{\infty}$, depending on $z$, such that $\left\{F^{n_{k}}(z)\right\}_{k=1}^{\infty} \subset Q_{-2}$ and

$$
\lim _{k \rightarrow \infty} \operatorname{Re}\left(F^{n_{k}}(z)\right)=-\infty .
$$


Fix $k \geq 1$ and consider the ball $B\left(z, K^{-1}\left|\left(F^{n_{k}}\right)^{\prime}(z)\right|^{-1}\right)$. Then

$$
B\left(z, K^{-1}\left|\left(F^{n_{k}}\right)^{\prime}(z)\right|^{-1}\right) \subset F_{z}^{-n_{k}}\left(B\left(F^{n_{k}}(z), 1\right)\right),
$$

where $F_{z}^{-n}: B\left(F^{n}(z), 1\right) \rightarrow \mathbb{C}$ is the analytic inverse branch of $F^{n}$ mapping $F^{n}(z)$ to $z$. Applying Koebe's distortion theorem and conformality of the measure $m$, we obtain

$$
\begin{aligned}
m\left(B\left(z, K^{-1}\left|\left(F^{n_{k}}\right)^{\prime}(z)\right|^{-1}\right)\right) & \leq K^{h}\left|\left(F^{n_{k}}\right)^{\prime}(z)\right|^{-h} m\left(B\left(F^{n_{k}}(z), 1\right)\right) \\
& \leq K^{2 h}\left(K^{-1}\left|\left(F^{n_{k}}\right)^{\prime}(z)\right|^{-1}\right)^{h} m\left(Q_{\operatorname{Re} F^{n_{k}}(z)+1}\right) .
\end{aligned}
$$

Since by (6.1) $\lim _{k \rightarrow \infty} m\left(Q_{\operatorname{Re} F^{n_{k}}(z)+1}\right)=0$, we see that

$$
\liminf _{r \rightarrow 0} \frac{m(B(z, r))}{r^{h}}=0 .
$$

Since $m\left(G \cap J_{r}(F)\right)>0$ for every nonempty open subset of $J_{r}(F)$, this implies (see an appropriate Converse Frostman's Type Theorem in [11, Theorem E(1), p. 58], or [23]) that $\mathrm{P}^{h}(G)=\infty$. Since $J_{r}(f)=$ $\bigcup_{k \in \mathbb{Z}}\left(J_{r}(F)+2 \pi i k\right)$, we are therefore done.

Theorem 6.2 We have $0<\mathrm{H}^{h}\left(J_{r}(F)\right)<\infty$.

Proof. Let $M>0$ be given by Theorem 4.10. Fix an integer $T \geq 1$ and a point $z \in J_{r, T}$. Consider the holomorphic inverse branches $F_{z}^{-n_{k}(z)}: B\left(y(z),(2 T)^{-1}\right) \rightarrow Q$ sending $F^{n_{k}(z)}(z)$ to $z$. Since, by $\frac{1}{4}$-Koebe's distortion theorem and the standard version of Koebe's distortion theorem,

$$
F_{z}^{-n_{k}(z)}\left(B\left(y(z), \frac{1}{2 T}\right)\right) \supset F_{z}^{-n_{k}(z)}\left(B\left(F^{n_{k}(z)}(z), \frac{1}{3 T}\right)\right) \supset B\left(z, \frac{1}{12 T}\left|\left(F^{n_{k}(z)}\right)^{\prime}(z)\right|^{-1}\right)
$$

and

$$
\begin{aligned}
F_{z}^{-n_{k}(z)}\left(B\left(y(z), \frac{1}{24 K T}\right)\right) & \subset F_{z}^{-n_{k}(z)}\left(B\left(F^{n_{k}(z)}(z), \frac{1}{12 K T}\right)\right) \\
& \subset B\left(z, \frac{1}{12 T}\left|\left(F^{n_{k}(z)}\right)^{\prime}(z)\right|^{-1}\right) .
\end{aligned}
$$

Using the conformality of the measure $m$ along with the standard version of Koebe's distortion theorem, and the fact that inf $\left\{m\left(B\left(w,(12 K T)^{-1}\right): w \in Q_{-2 T}^{c}\right\}>0\right.$, we deduce that

$$
B_{T}^{-1} r_{k}(z)^{h} \leq \nu\left(B\left(z, r_{k}(z)\right)\right) \leq B_{T} r_{k}(z)^{h}
$$

where $r_{k}(z)=(12 T)^{-1}\left|\left(F^{n_{k}(z)}\right)^{\prime}(z)\right|^{-1}$ and $B_{T}$ is independent of $z$ and $k$. It follows from (6.2) that $\left.\mathrm{H}^{h}\right|_{J_{r, T}(F)} \prec m$ for every $T \geq 1$ and that $\mathrm{H}^{h}\left(J_{r, M}(F)\right)<\infty$. Since $m\left(J_{r, T}(F) \backslash J_{r, M}(F)\right) \leq m\left(J_{r}(F) \backslash\right.$ $\left.J_{r, M}(F)\right)=0$ and since $J_{r}(F)=\bigcup_{n=0}^{\infty} J_{r, M+n}(F)$, we therefore conclude that $\mathrm{H}^{h}\left(J_{r}(F)\right)=\mathrm{H}^{h}\left(J_{r, M}(F)\right)<$ $\infty$.

We shall prove now that $\mathrm{H}^{h}\left(J_{r}(F)\right)>0$. Put

$$
\kappa=\min \left\{1, R, \frac{\lambda}{2} e^{-2 T_{2}}\right\} \text {. }
$$

Fix $z \in J_{r}(F)$ and $r \in\left(0, \kappa\left(2^{8} K\right)^{-1}\right)$. Since, by Lemma 4.11, $\limsup _{n \rightarrow \infty}\left|\left(f^{n}\right)^{\prime}(z)\right|=+\infty$, there exists a least $n=n(z, r) \geq-1$ such that

$$
r\left|\left(f^{n+1}\right)^{\prime}(z)\right|>\kappa\left(2^{8} K\right)^{-1}
$$

Thus $n \geq 0$ and

$$
r\left|\left(f^{n}\right)^{\prime}(z)\right| \leq \kappa\left(2^{8} K\right)^{-1} .
$$


Suppose that the holomorphic inverse branch of $f^{n}$ defined on $B\left(f^{n}(z), 32 r\left|\left(f^{n}\right)^{\prime}(z)\right|\right)$ and sending $f^{n}(z)$ to $z$ does not exist. Then $n \geq 1$ and let $1 \leq k \leq n$ be the largest integer such that the holomorphic inverse branch of $f^{n-(k-1)}$ defined on $B\left(f^{n}(z), 32 r\left|\left(f^{n}\right)^{\prime}(z)\right|\right)$ and sending $f^{n}(z)$ to $f^{k-1}(z)$ does not exist. This implies that $0 \in f_{k}^{-(n-k)}\left(B\left(f^{n}(z), 32 r\left|\left(f^{n}\right)^{\prime}(z)\right|\right)\right)$, where $f_{k}^{-(n-k)}: B\left(f^{n}(z), 32 r\left|\left(f^{n}\right)^{\prime}(z)\right|\right) \rightarrow \mathbb{C}$ is the holomorphic inverse branch of $f^{n-k}$ sending $f^{n}(z)$ to $f^{k}(z)$. In addition, $n=k$ since $0 \notin f^{-1}(\overline{\mathbb{C}})$. Hence $\left|f^{n}(z)\right|<32 K r\left|\left(f^{n}\right)^{\prime}(z)\right| \leq \kappa \leq R$. It now follows from (A.14) that

$$
\operatorname{Re}\left(f^{n-1}(z)\right) \leq \frac{1}{2} \log \left(\frac{2}{\lambda}\right)+\frac{1}{2} \log \kappa \leq-T_{2}
$$

We therefore get by (A.2) and (A.5) that $\left|f^{\prime}\left(f^{n-1}(z)\right)\right| \leq 4 \lambda \exp \left(2 \operatorname{Re}\left(f^{n-1}(z)\right)\right) \leq 8\left|f^{n}(z)\right|$. Consequently, since $r\left|\left(f^{n-1}\right)^{\prime}(z)\right|<\kappa\left(2^{8} K\right)^{-1}$, we conclude that

$$
\begin{aligned}
32 K r\left|\left(f^{n}\right)^{\prime}(z)\right| & =32 K r\left|\left(f^{n-1}\right)^{\prime}(z)\right| \cdot\left|f^{\prime}\left(f^{n-1}(z)\right)\right| \\
& \leq 32 K r\left|\left(f^{n-1}\right)^{\prime}(z)\right| \cdot 8\left|f^{n}(z)\right| \\
& =2^{8} K r\left|\left(f^{n-1}\right)^{\prime}(z)\right| \cdot\left|f^{n}(z)\right| \\
& <\left|f^{n}(z)\right| .
\end{aligned}
$$

This contradiction shows that the holomorphic inverse branch $f_{z}^{-n}: B\left(f^{n}(z), 32 r\left|\left(f^{n}\right)^{\prime}(z)\right|\right) \rightarrow \mathbb{C}$ of $f^{n}$ sending $f^{n}(z)$ to $z$, is well-defined. Now, the map $f$ restricted to $B\left(f^{n}(z), 32 r\left|\left(f^{n}\right)^{\prime}(z)\right|\right)$ is 1-to-1, and by Koebe's $\frac{1}{4}$-Theorem

$$
f\left(B\left(f^{n}(z), 32 r\left|\left(f^{n}\right)^{\prime}(z)\right|\right)\right) \supset B\left(f^{n+1}(z), 8 r\left|\left(f^{n+1}\right)^{\prime}(z)\right|\right) .
$$

Hence there exists a unique holomorphic inverse branch $f_{z}^{-(n+1)}: B\left(f^{n+1}(z), 8 r\left|\left(f^{n+1}\right)^{\prime}(z)\right|\right) \rightarrow \mathbb{C}$ of $f^{n+1}$ mapping $f^{n+1}(z)$ to $z$. Applying Koebe's $\frac{1}{4}$-Theorem again, we see that

$$
f_{z}^{-(n+1)}\left(B\left(f^{n+1}(z), 4 r\left|\left(f^{n+1}\right)^{\prime}(z)\right|\right)\right) \supset B(z, r) .
$$

Since the ball $B\left(f^{n+1}(z), 4 r\left|\left(f^{n+1}\right)^{\prime}(z)\right|\right)$ intersects at most $\frac{1}{\pi} 4 r\left|\left(f^{n+1}\right)^{\prime}(z)\right|+2 \preceq r\left|\left(f^{n+1}\right)^{\prime}(z)\right|$ horizontal strips of the form $2 \pi i j+(\mathbb{R} \times[0,2 \pi)), j \in \mathbb{Z}$, using (6.4), Koebe's Distortion Theorem, $h$-conformality of the measure $m$ and, at the end, (6.3), we get

$$
\begin{aligned}
r^{-h}(m(B(z, r)) & \preceq r^{-h} K^{h}\left|\left(f^{n+1}\right)^{\prime}(z)\right|^{-h}\left(r\left|\left(f^{n+1}\right)^{\prime}(z)\right|\right) m\left(\Pi\left(B\left(f^{n+1}(z), 4 r\left|\left(f^{n+1}\right)^{\prime}(z)\right|\right)\right)\right) \\
& \leq r^{-h} K^{h}\left|\left(f^{n+1}\right)^{\prime}(z)\right|^{-h}\left(r\left|\left(f^{n+1}\right)^{\prime}(z)\right|\right) \\
& =K^{h}\left(r\left|\left(f^{n+1}\right)^{\prime}(z)\right|\right)^{1-h} \\
& <K^{h}\left(2^{8} K\right)^{h-1} .
\end{aligned}
$$

We are done by applying an appropriate Converse Frostman's Type Theorem (see e.g. [11, Theorem D(2)] or [23]).

We end this section with the following.

Theorem 6.3 We have $\operatorname{HD}\left(J_{r}(f)\right)=\operatorname{HD}\left(J_{r}(F)\right)=h \in(1,2)$.

Proof. The first equality sign is obvious. The second one is an immediate consequence of Theorem 6.2. The inequality $h>1$ has been established in Theorem 4.8. We are left to show that $h<2$. And indeed, suppose on the contrary that $h=2$. Then $\mathrm{P}^{h}=\mathrm{P}^{2}$ is a constant multiple of the two-dimensional Lebesgue measure on $\mathbb{C}$ and we have a contradiction with Theorem 6.1 . 


\section{A Appendix: Useful formulas}

It follows from (1.1) that

$$
f^{\prime}(z)=\frac{-2 \lambda}{\left(e^{z}-e^{-z}\right)^{2}} .
$$

By (A.1) there exists an integer $T_{1}>0$ so large that

$$
\lambda e^{2 \operatorname{Re}(z)} \leq\left|f^{\prime}(z)\right| \leq 4 \lambda e^{2 \operatorname{Re}(z)}
$$

for all $z \in \mathbb{C}_{-T_{1}}$. Since $\lim _{z \rightarrow 0} \frac{e^{z}-e^{-z}}{z}=2$, it follows from (A.1) that there exists $R>0$ so small that if $|z| \leq R$, then

and

$$
\frac{\lambda}{4|z|} \leq|f(z)| \leq \frac{\lambda}{|z|}
$$

$$
\frac{\lambda}{4}|z|^{-2} \leq\left|f^{\prime}(z)\right| \leq \lambda|z|^{-2}
$$

It immediately follows from the formula defining $f$ that there exists an integer $T_{2} \geq T_{1}$ such that

$$
\frac{\lambda}{2} e^{2 \operatorname{Re}(z)} \leq|f(z)| \leq 2 \lambda e^{2 \operatorname{Re}(z)}
$$

for all $z \in \mathbb{C}_{-T_{2}}$. It in turn immediately follows from this formula that there exists an integer $T_{3} \geq T_{2}$ such that

$$
f\left(\mathbb{C}_{-T_{3}}\right) \subset B(0, R) .
$$

Using the Chain Rule and combining (A.2) along with (A.4)-(A.6), we see that for all $z \in \mathbb{C}_{-T_{3}}$

$$
\begin{aligned}
\frac{1}{16} e^{-2 \operatorname{Re}(z)} & =\frac{\lambda^{2}}{4} e^{2 \operatorname{Re}(z)}(2 \lambda)^{-2} e^{-4 \operatorname{Re}(z)} \leq \frac{\lambda^{2}}{4} e^{2 \operatorname{Re}(z)}|f(z)|^{-2} \leq\left|\left(f^{2}\right)^{\prime}(z)\right| \\
& \leq 4 \lambda^{2} e^{2 \operatorname{Re}(z)}|f(z)|^{-2} \leq 4 \lambda^{2} 4 \lambda^{-2} e^{2 \operatorname{Re}(z)} e^{-4 \operatorname{Re}(z)}=16 e^{-2 \operatorname{Re}(z)}
\end{aligned}
$$

It follows from this and (A.4) that

$$
\lim _{\operatorname{Re}(z) \rightarrow-\infty}\left|\left(f^{2}\right)^{\prime}(z)\right|=\infty
$$

and

$$
\lim _{\operatorname{Re}(z) \rightarrow 0}\left|f^{\prime}(z)\right|=\infty
$$

Notice that $f^{-1}\left(\mathbb{C}_{0}\right) \subset \mathbb{C}_{0}$ and $\mathbb{C}_{0}$ contains no asymptotic values $(0$ and $\lambda)$. So, all the holomorphic inverse branches of $f$ are well-defined on $\mathbb{C}_{0}$ and given by the formulas:

$$
f_{k}^{-1}(z)=-\frac{1}{2} \log \left|1-\frac{\lambda}{z}\right|-\left(\frac{1}{2} \operatorname{Arg}\left(1-\frac{\lambda}{z}\right)+k \pi\right) i
$$

$k \in \mathbb{Z}$, where $\operatorname{Arg} w$ is the principal value of the $\operatorname{argument}$ i.e., $\operatorname{Arg} w \in[-\pi, \pi)$. Equivalently

$$
f_{k}^{-1}(z)=-\frac{1}{2} \log _{k}\left(1-\frac{\lambda}{z}\right)
$$

where the branch of logarithm $\log _{k}$ is determined by the requirement that $\log _{k}(1)=2 \pi i k$. Hence

$$
\left(f_{k}^{-1}\right)^{\prime}(z)=\frac{-\lambda}{2 z(z-\lambda)}
$$


For $R>0$ sufficiently small and all $z \in B_{-}(0, R)$, we have

$$
\frac{2 \lambda}{|z|} \geq 1+\left|\frac{\lambda}{z}\right| \geq\left|1-\frac{\lambda}{z}\right| \geq\left|\frac{\lambda}{z}\right|-1 \geq \frac{\lambda}{2|z|} .
$$

So

$$
\log 2+\log \lambda-\log |z| \geq \log \left|1-\frac{\lambda}{z}\right| \geq \log \lambda-\log 2-\log |z| .
$$

Thus, if $R>0$ and $z \in B_{-}(0, R)$ then for every $k \in \mathbb{Z}$

and

$$
\operatorname{Re}\left(f_{k}^{-1}(z)\right)=-\frac{1}{2} \log \left|1-\frac{\lambda}{z}\right| \leq \frac{1}{2} \log 2-\frac{1}{2} \log \lambda+\frac{1}{2} \log |z| \leq c_{1}+\frac{1}{2} \log R
$$

$$
\operatorname{Re}\left(f_{k}^{-1}(z)\right) \geq c_{2}+\frac{1}{2} \log |z|
$$

where $c_{1}=\frac{1}{2} \log 2-\frac{1}{2} \log \lambda$ and $c_{2}=-\frac{1}{2}(\log 2+\log \lambda)$. Therefore,

$$
f_{0}^{-1}\left(B_{-}(0, R)\right) \subset P_{c_{1}+\frac{1}{2} \log R}:=\left\{z \in \mathbb{C}: \operatorname{Re}(z)<c_{1}+\frac{1}{2} \log R,-\frac{\pi}{2}<\operatorname{Im}(z) \leq \frac{\pi}{2}\right\} .
$$

It follows from (A.15) and (A.16) that if $0<r<R$ then

$$
f_{0}^{-1}\left(A_{-}(0, r, R)\right) \subset P_{c_{1}+\frac{1}{2} \log R} \backslash P_{c_{2}+\frac{1}{2} \log r} .
$$

It follows from (A.11) that

$$
\frac{\lambda}{4|z|} \leq\left|f_{0}^{-1}(z)\right| \leq \frac{\lambda}{|z|}
$$

for some $T_{4} \geq T_{3}$ and all $z$ with $|z| \geq T_{4}$. Recall that the dynamics of $F$ is closely related to the dynamics of $f$, since $F^{n}(z)=\Pi\left(f^{n}(z)\right)$ for all $n \geq 0$. Notice that

$$
\begin{aligned}
& \left.J(f) \subset \mathbb{C}_{0} \cup\{\pi k i: k \in \mathbb{Z}\}, \quad J(F)=\Pi(J(f)), \quad F(J(F)) \subset J(F) \cup\{\infty\}\right) \\
& \operatorname{dist}(J(F), \Pi(\{z \in \mathbb{C}: \operatorname{Im} z=\pi / 2\}))>0
\end{aligned}
$$

and

$$
J(F) \cap \Pi\left(\mathbb{C}_{-T_{5}}\right) \subset\left\{z \in \Pi\left(\mathbb{C}_{-T_{5}}\right):|\operatorname{Im} z|<\frac{\pi}{3}\right\}
$$

with $T_{5} \geq T_{4}$ sufficiently large.

Acknowledgements We are very grateful for the referee of our article for his constructive remarks and suggestions, which influenced the final exposition of our work.

The research of the first named author was supported in part by the Polish KBN Grant No. 2 PO3A 03425 and the Warsaw University of Technology Grant No. 504G 11200043000.

The research of the second named author was supported in part by the NSF Grant DMS 0100078. He also wishes to thank the Mathematical Institute of the Polish Academy of Sciences, where a part of this research was conducted.

The research of both authors was supported in part by the NSF/PAN grant INT-0306004.

\section{References}

[1] I. N. Baker, J. Kotus, and Y. Lü, Iterates of meromorphic functions I, Ergodic Theory Dynam. Systems 11, 241-248 (1991).

[2] I. N. Baker, J. Kotus, and Y. Lü, Iterates of meromorphic functions II: Examples of wandering domains, J. London Math. Soc. (2) 42, 267-278 (1990).

[3] I. N. Baker, J. Kotus, and Y. Lü, Iterates of meromorphic functions III: Preperiodic domains, Ergodic Theory Dynam. Systems 11, 603-618 (1991). 
[4] I. N. Baker, J. Kotus, and Y. Lü, Iterates of meromorphic functions IV, Results Math. 22, 651-656 (1992).

[5] W. Bergweiler, Iteration of meromorphic functions, Bull. Amer. Math. Soc. (N.S.) 29:2, 151-188 (1993).

[6] P. Billingsley, Convergence of Probability Measures, 2nd Edition (Wiley, 1999).

[7] H. Bock, Über das Iterationsverhalten meromorpher Funktionen auf der Juliamenge, Ph.D. thesis, University Achen (1998).

[8] M. Denker and M. Urbański, Hausdorff and conformal measures on Julia sets with a rationally indifferent periodic point, J. London Math. Soc. (2) 43, 107-118 (1991).

[9] M. Denker and M. Urbański, On the existence of conformal measures, Trans. Amer. Math. Soc. 328, 563-587 (1991).

[10] M. Denker and M. Urbański, On Sullivan's conformal measures for rational maps of the Riemann sphere, Nonlinearity 4, 365-384 (1991).

[11] M. Denker and M. Urbański, Geometric measures for parabolic rational maps, Ergodic Theory Dynam. Systems 12 , 53-66 (1992).

[12] R. Devaney and L. Keen, Dynamics of meromorphic maps with polynomial Schwarzian derivative, Ann. Sci. Ecole Norm. Sup. (4) 22, 55-81 (1989).

[13] H. F. Federer, Geometric Measure Theory (Springer-Verlag, New York, 1969).

[14] M. Guzmán, Differentiation of Integrals in $\mathbb{R}^{n}$, Lecture Notes in Mathematics Vol. 481 (Springer-Verlag).

[15] J. Kotus, On the Hausdorff dimension of the Julia sets of meromorphic functions I, Bull. Soc. Math. France 122, 305-331 (1995).

[16] J. Kotus and M. Urbański, Existence of invariant measures for transcendental subexpanding functions, Math. Z. 243, 25-36 (2003).

[17] J. Kotus and M. Urbański, Conformal, geometric and invariant measures for transcendental expanding functions, Math. Ann. 324, 619-656 (2002).

[18] J. Kotus and M. Urbański, Hausdorff dimension and Hausdorff measures of Julia sets of elliptic functions, Bull. London Math. Soc. 35, 269-275 (2003).

[19] J. Kotus and M. Urbański, Geometry and ergodic theory of non-recurrent elliptic functions, J. Anal. Math. 93, 35-102 (2004).

[20] M. Martens, The existence of $\sigma$-finite invariant measures, applications to real one-dimensional dynamics, Front for the Mathematics ArXiv, http://front.math.ucdavis.edu/; math.DS/9201300.

[21] R. L. Mauldin and M. Urbański, Dimensions and measures in infinite iterated function systems, Proc. London Math. Soc. (3) 73, 105-154 (1996).

[22] J. Milnor, Dynamics in one Complex Variable, Introductory Lectures (Vieweg, 2000).

[23] F. Przytycki and M. Urbański, Fractals in the Plane-Ergodic Theory Methods, available on Urbański's webpage, to be published by Cambridge Univ. Press.

[24] P. J. Rippon and G. M. Stallard, Iteration of a class of hyperbolic meromorphic functions, Proc. Amer. Math. Soc. 127, 3251-3258 (1999).

[25] S. J. Taylor and C. Tricot, Packing measure, and its evaluation for a Brownian path, Trans. Amer. Math. Soc. 288, 679-699 (1985).

[26] M. Urbański, On Hausdorff dimension of Julia set with a rationally indifferent periodic point, Studia Math. 97, 167-188 (1991).

[27] M. Urbański, Rational functions with no recurrent critical points, Ergodic Theory Dynam. Systems 14, 391-414 (1994).

[28] M. Urbański, Geometry and ergodic theory of conformal non-recurrent dynamics, Ergodic Theory Dynam. Systems 17, 1449-1476 (1997).

[29] M. Urbański and A. Zdunik, The finer geometry and dynamics of exponential family, Michigan Math. J. 51, 227-250 (2003).

[30] M. Urbański and A. Zdunik, Real analyticity of Hausdorff dimension of finer Julia sets of exponential family, Ergodic Theory Dynam. Systems 24, 279-315 (2004).

[31] M. Urbański and A. Zdunik, The parabolic map $f_{1 / e}(z)=\frac{1}{e} e^{z}$, Indag. Math. (N.S.) 15, 419-433 (2004).

[32] M. Urbański and A. Zdunik, Geometry and ergodic theory of non-hyperbolic exponential maps, Trans. Amer. Math. Soc., to appear. 